\title{
QUALIDADE DE VIDA DOS PROFISSIONAIS ATUANTES NO CENTRO CIRÚRGICO EM PATOS DE MINAS - MG ${ }^{1}$
}

\author{
Luciana Maria Pereira Soares ${ }^{2}$ \\ Victor Constante Oliveira ${ }^{3}$ \\ Luíza Araújo AmâncioSousa ${ }^{4}$
}

\begin{abstract}
RESUMO
Este estudo descritivo exploratório teve por objetivo avaliar os fatores estressantes que interferem na qualidade de vida dos profissionais que atuam no CC em Patos de Minas-MG. Os dados foram coletados entre os meses de fevereiro e março de 2017, por meio de um questionário contendo uma questão norteadora com 22 profissionais, sendo 13 técnicos de enfermagem, cinco enfermeiros e quatro médicos. Aplicou-se análise de conteúdo temática resultando em seis categorias.Os resultados apontam que a maioria era do sexo feminino, casados, com idade média entre 26 e 45 anos, e técnicos de enfermagem. Na avaliação geral da qualidade de vida, os dados obtidos revelaram que o fator que mais gera estresse na vida desses profissionais é o tipo de ambiente fechado, sendo que os demais fatores variaram de acordo com a categoria profissional. Conclui-se que os profissionais de enfermagem vivenciaram o estresse relacionado a diversos fatores de natureza objetiva.
\end{abstract}

Palavras-chave: Saúde ocupacional. Condições de trabalho. Fatores estressores. Centro cirúrgico hospitalar.

\begin{abstract}
This exploratory descriptive study aimed to evaluate the stressors that interfere in the quality of life of the professionals who work in the CC in Patos de Minas-MG.The data were collected between February and March 2017, through a questionnaire containing a guiding question with 22 professionals, 13 nursing technicians, five nurses and four doctors.Thematic content analysis was applied resulting in six categories.The results indicate that the majority were female, married, with an average age between 26 and 45 years, and nursing technicians.In general evaluation of the quality of life, the data obtained revealed that the factor that causes the most stress in the life of these professionals is the type of closed environment, and the other factors varied according to the professional category.It was concluded that the nursing professionals experienced the stress related to several objective factors.
\end{abstract}

Keywords: Occupational health. Work conditions. Stressors. Hospital surgical center.

\footnotetext{
${ }^{1}$ Artigo científico apresentado à disciplina de Trabalho de Conclusão de Curso (TCC) da Faculdade Patos de Minas (FPM) como requisito para conclusão do curso de enfermagem.

${ }^{2}$ Graduanda em Enfermagem pela Faculdade Patos de Minas - FPM.

${ }^{3}$ Mestre em Genética e Bioquímica pela Universidade Federal de Uberlândia - UFU.

${ }^{4}$ Mestre em Gestão Organizacional pela Universidade Federal do Goiás - UFG, e docente do curso de enfermagem da Faculdade Patos de Minas-FPM.
} 


\section{INTRODUÇÃo}

0 centro cirúrgico (CC) é um setor hospitalar que conta com uma equipe multiprofissional atuante como: médicos anestesiologistas, médicos cirurgiões, instrumentadorescirúrgicos, enfermeiro coordenador e técnicos de enfermagem; equipamentos e instrumentos apropriados para a realização do processo cirúrgico, oferecendo condições que proporcionam qualidade e segurança para os profissionais de enfermagem e para que atendam as necessidades dos pacientes antes, durante e após a cirurgia (RODRIGUES e SOUZA, 1993; MARTINS, 2013).

O CC écaracterizado como uma área física fechada, de risco e com vários regulamentos e rotinas (STUMM, MAÇALAI e KIRCHNER, 2006). Os procedimentos de trabalho dependem da interação entre os profissionais que atuam na referida unidade, devendo acontecer de forma harmoniosa (CAREGNATO, 2002), além de apresentar conhecimento científico sobre os procedimentos cirúrgicos, responsabilidade, habilidade técnica, e estabilidade emocional (STUMM, MAÇALAI e KIRCHNER, 2006), pois estes profissionais têm tolerado jornadas de trabalho cada vez mais prolongadas dentro desse setor, com proporção inadequada de pacientes por profissionais qualificados, turnos alternados, baixa remuneração, exposição a substâncias tóxicas e fatores de risco relacionados ao ambiente, levando a uma circunstância conhecida como sobrecarga de trabalho (OLER et al.,2005).

Segundo Ferrareze, Ferreira e Carvalho (2006), o estresse ocupacional, ao qual trabalhador moderno está exposto, tem se destacado como assunto de suma importância, principalmente em algumas profissões como, por exemplo, os profissionais que atuam no CC. 0 estresse ocupacional é um estado em que ocorre desgaste anormal do organismo humano e/ou diminuição da capacidade de trabalho, devido basicamente à incapacidade prolongada de o indivíduo tolerar, superar ou se adaptar às exigências de natureza física e emocional existentes em seu ambiente de trabalho ou de vida (SCHMIDT et al., 2009).

Desta forma, a exposição aos riscos físicos, químicos, biológicos, ergonômicos e asobrecarga de trabalho aumenta cada vez mais o nível de estresse dos profissionais, pois o CC exige um alto grau de responsabilidade, além de o profissional permanecer nas salas de cirurgia por um longo tempo, acompanhando e atuando nos diversos procedimentos cirúrgicos, de diferentes doenças e com grau de complexidade variado, desde os mais simples aos mais graves.Tais condições resultam no (des)equilíbrio físico e emocional, insatisfação, com consequente queda da produtividade, e acidentes de trabalho (MIRANDA, 2006). Diante disso, o objetivo do presente estudo foi avaliar quais os fatoresestressantes que interferem na qualidade de vida dos profissionais que atuam no Centro Cirúrgico em Patos de Minas - MG. 


\section{MATERIAL E MÉTODOS}

\subsection{Tipo de estudo}

Trata-se de um estudo exploratório, descritivo e transversal sobre a avaliação dos fatores que interferem na qualidade de vida da equipe de profissionaisatuantesno centro cirúrgico na cidade dePatos de Minas.

0 estudo de natureza descritiva-exploratório permite ao pesquisador detalhar e analisar um determinado problema propiciando a esse maior conhecimento em torno do tema, além de definir objetivos e buscar maiores informações sobre determinado assunto estudado (CERVO; BERVIAN, 1996).

\subsection{Local da pesquisa}

0 presente estudo foi desenvolvido em Centros Cirúrgicos de diferentes hospitais, por compreender de um setor que possui características próprias de trabalho.Os encontros com os profissionais aconteceram após as sessões de cirurgia, mantendo a privacidade e conforto para os sujeitos.

\subsection{Sujeitos da pesquisa}

Fizeram parte do estudo vinte e doissujeitos $(n=22)$, incluindo técnicos de enfermagem, enfermeiros e médicos, os quais foram selecionados de forma aleatória, no período de fevereiro e março de 2017, por atenderem aos seguintes critérios de seleção: trabalhar no centro cirúrgico hámais de dois anos,ter jornada de trabalho igual ou superior a 44 horas semanais, ter idade maior que 18 anos, manifestar interesse em falar sobre o tema proposto, aceitar formalmente participar da pesquisae assinar o TCLE ( Termo de consentimento Livre Esclarecido).

\subsection{Coleta de dados}

Os dados foram obtidos por meio de entrevistas comuma questão norteadora:"Para você, quais são os fatores que geram estresse durante o seu trabalho no centro cirúrgico?".Esta pergunta por sua vez, visouobter dados quanto à percepção da equipe quanto à exposição dos fatores estressantes. 


\subsection{Análise de dados}

Os dados foram analisados a partir da leitura da questão norteadora, e seguiramos seguintes passos:codificação dos dados com a identificação dos trechos que abordavam a questão pesquisada; formação das categorias temáticas; elaboração da identificação do conjunto de expressões que representam os fatores estressantes aos quais a equipe do centro cirúrgico está exposta durante sua jornada de trabalho.

\subsection{Aspectos éticos da pesquisa}

O projeto da pesquisa foi apreciado pelo Comitê de Ética da Faculdade Patos de Minas - FPM e aprovado pelo protocolo do CEP: no 8078/2017, CAAE: 67667417.9.0000.8078. Além disso, foi considerado o princípio da autonomia e respeitada a privacidade, principalmente no que se refere ao Termo de Consentimento Livre e Esclarecido (TCLE), instrumento indispensável para que se possam realizar pesquisas que envolvem seres humanos.

\section{RESULTADOS}

Os dados coletados foram categorizados segundo objetivo da pesquisa, buscando-se agrupar as respostas semelhantes. Os resultados foram analisados quanti-qualitativamente e apresentados em forma de figuras, tabelas e discursiva. Para compor a amostra do estudo foram entrevistados vinte e dois sujeitos $(n=22)$ atuantes no centro cirúrgico (CC) em diferentes hospitais de Patos de Minas-MG que possuíam os seguintes vínculos empregatícios: Técnicos de enfermagem, enfermeiros e médicos (Figura 1). Durante o período estabelecido para coleta de dados foram excluídos os trabalhadores de férias, licença médica e licença gestante.

Conforme pode ser observado na Figura 1, os participantes do estudo eram predominantemente Técnicos de enfermagem (59\%), sendo que as demais proporções de enfermeiros $(22,8 \%)$ e médicos $(18,2 \%)$ foram semelhantes.

FIGURA 1: Distribuição total dos profissionais entrevistados que atuam no Centro Cirúrgico em diferentes hospitais de Patos de Minas-MG. 


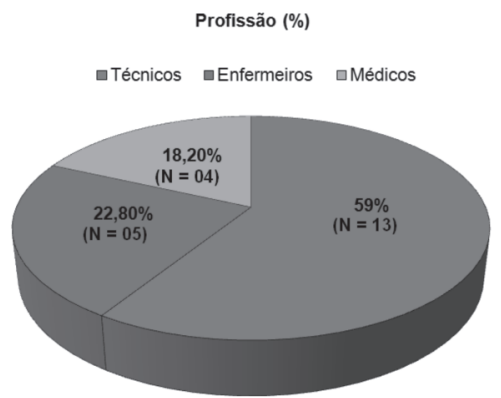

Os resultados foram analisados em função da amostra estudada $(n=22)$, agrupados de forma a atender os objetivos deste estudo. A descrição dos sujeitos entrevistados quanto ao sexo, faixa etária, estado civil, grau de instrução e remuneração é apresentada na Tabela 1.

TABELA 1- Descrição das variáveis sociodemográficas da amostra avaliada (n=22). Patos de Minas, MG, 2017.

\begin{tabular}{|c|c|}
\hline VARIÁVELN & $(\%)$ \\
\hline \multicolumn{2}{|l|}{ Sexo } \\
\hline Masculino & $06(27,2)$ \\
\hline Feminino & $16(72,8)$ \\
\hline \multicolumn{2}{|l|}{ Faixa Etária } \\
\hline 18 a 25 anos & $02(9,0)$ \\
\hline 26 a 35 anos & $08(36,4)$ \\
\hline 36 a 45 anos & $08(36,4)$ \\
\hline Acima de 45 anos & $04(18,2)$ \\
\hline \multicolumn{2}{|l|}{ Estado Civil } \\
\hline Casado & $17(77,3)$ \\
\hline Solteiro & $03(13,7)$ \\
\hline Separado & $02(9,0)$ \\
\hline Viúvo & $00(0,0)$ \\
\hline Outros & $00(0,0)$ \\
\hline \multicolumn{2}{|l|}{ Formação } \\
\hline $2^{\circ}$ Grau completo & $08(36,4)$ \\
\hline Superior incompleto & $06(27,2)$ \\
\hline Superior completo & $08(36,4)$ \\
\hline \multicolumn{2}{|l|}{ Faixa Salarial } \\
\hline $\mathrm{R} \$ 1200,00$ a $\mathrm{R} \$ 1999,00$ & $07(31,8)$ \\
\hline $\mathrm{R} \$ 2000,00$ a $\mathrm{R} \$ 2999,00$ & $06(27,2)$ \\
\hline $\mathrm{R} \$ 3000,00$ a $\mathrm{R} \$ 3999,00$ & $04(18,2)$ \\
\hline $\mathrm{R} \$ 4000,00$ a $\mathrm{R} \$ 4999,00$ & $01(4,6)$ \\
\hline Acima de $\mathrm{R} \$ 5000,00$ & $04(18,2)$ \\
\hline Total & $22(100)$ \\
\hline
\end{tabular}


Conforme apresentado na Tabela 1, os participantes do estudo eram predominantemente do sexo feminino $(72,8 \%)$, com idade compreendida entre $26 \mathrm{e}$ 45 anos (72,8\%). Além disso, a média geral de faixa etária foi de36 anos. Quanto ao estado civil dos profissionais, $17(77,3 \%)$ eram casados, três $(13,7 \%)$ solteiros, dois $(9,0 \%)$ estavam separados dos cônjuges e não teve viúvos. Em relação à escolaridade dos profissionais, oito $(36,4 \%)$ possuíam o $2^{\circ}$ grau completo, seis $(27,2 \%)$ superior incompleto, e oito $(36,4 \%)$ superior completo, sendo que a maioria tem remuneração entre $R \$ 1200,00$ e $R \$ 2999,00$.

Como descrito na nossa metodologia, à qualidade de vida dos profissionais que atuam no CC, e os fatores que geram estresse nesse ambiente de trabalho foi avaliado por meio da questão norteadora presente nosquestionários. A análise das falas levou a construção de seis categorias temáticas que mais geram estresse no cotidiano desses profissionais: Relações interpessoais com a equipe multiprofissional, e com os familiares dos pacientes; Falta de materiais e equipamentos, incluindo os estragos que impossibilitam a utilização; Tipo de ambiente Fechado, no qual estão sujeitos a diversos procedimentos cirúrgicos de baixa, média e alta complexidade, incluindo as situações de emergência; Falta de assiduidade e pontualidade, tanto dos profissionais como dos pacientes; Baixa remuneração e desvalorização; e sobrecarga de trabalho.

Vale ressaltar que por se tratar de uma pergunta discursiva, cada sujeito entrevistado pode escrever várias respostas, inclusive mais de uma resposta relacionada a uma mesma categoria temática. Assim, as respostas que se enquadraram na mesma categoria, foram reunidas.A Tabela 2 Apresenta a proporção dos fatores geradores de estresse no CC distribuídos nas seis categorias e separados por profissão: Técnicos, enfermeiros e médicos.

TABELA 2- Descrição das variáveis geradoras de estresse nos profissionais atuantes no Centro Cirúrgico $(n=22)$, separados por profissão. Patos de Minas, MG, 2017.

\begin{tabular}{lccc}
\hline \multicolumn{1}{c}{ Fator } & $\begin{array}{c}\text { Técnicos } \\
\mathbf{n ~ ( \% )}\end{array}$ & $\begin{array}{c}\text { Enfermeiros } \\
\mathbf{n ~ ( \% )}\end{array}$ & $\begin{array}{c}\text { Médicos } \\
\mathbf{n ~ ( \% )}\end{array}$ \\
\hline $\begin{array}{l}\text { Relações } \\
\text { interpessoais }\end{array}$ & $8(19,0)$ & $6(24,0)$ & $2(13,3)$ \\
$\begin{array}{l}\text { Falta de Materiais e } \\
\text { Equipamentos }\end{array}$ & $4(9,5)$ & $4(16,0)$ & $3(20,0)$ \\
$\begin{array}{l}\text { Tipo de ambiente } \\
\text { fechado }\end{array}$ & $13(31,0)$ & $6(24,0)$ & $6(40,0)$ \\
$\begin{array}{l}\text { Falta de assiduidadee } \\
\text { pontualidade }\end{array}$ & $5(11,9)$ & $4(16,0)$ & $3(20,0)$ \\
& & &
\end{tabular}




\begin{tabular}{lccc}
\hline \multicolumn{1}{c}{ Fator } & $\begin{array}{c}\text { Técnicos } \\
\mathbf{n}(\%)\end{array}$ & $\begin{array}{c}\text { Enfermeiros } \\
\mathbf{n}(\%)\end{array}$ & $\begin{array}{c}\text { Médicos } \\
\mathbf{n}(\%)\end{array}$ \\
\hline $\begin{array}{l}\text { Baixa remuneração e } \\
\text { desvalorização }\end{array}$ & $0(0,0)$ & $1(4,0)$ & $0(0,0)$ \\
$\begin{array}{l}\text { Sobrecarga de } \\
\text { trabalho }\end{array}$ & $12(28,6)$ & $4(16,0)$ & $1(6,7)$ \\
Total $^{*}$ & $\mathbf{4 2 ( 1 0 0 )}$ & $\mathbf{2 5 ( 1 0 0 )}$ & $\mathbf{1 5 ( 1 0 0 )}$ \\
\hline
\end{tabular}

$\mathrm{n}=$ Número de respostas por grupo de fatores geradores de estresse.

* Considerando mais de uma resposta por sujeito entrevistado.

Como pode ser observado na Tabela 2, o tipo de ambiente fechado foi o fator gerador de estresse mais citado por ambos profissionais: Técnicos (31\%), Enfermeiros (24\%) e médicos (40\%). Os demais fatores variaram de acordo com a categoria profissional. Com relação aos técnicos de enfermagem, o segundo fator que mais gera estresse é a sobrecarga de trabalho (28,6\%), seguido das relações interpessoais (19\%), falta de assiduidade e pontualidade (11,9\%), e falta de materiais e equipamentos $(9,5 \%)$, não tendo nenhuma citação referente à baixa remuneração e desvalorização. Em relação aos enfermeiros, as relações interpessoais apresentaram a mesma proporção do tipo de ambiente (24\%), seguido da falta de materiais e equipamentos, falta de assiduidade e pontualidade e sobrecarga de trabalho (16\%), e baixa remuneração e desvalorização (4\%). Quanto aos médicos, os outros fatores que mais causam estresse é a falta de materiais e equipamentos e falta de assiduidade e pontualidade (20\%), seguido das relações interpessoais $(13,3 \%)$ e sobrecarga de trabalho $(6,7 \%)$, não tendo nenhuma citação referente à baixa remuneração e desvalorização.

A comparação entre os seis grupos de fatores geradores de estresse e as categorias de profissionais pode melhor ser observada na Figura 2.

FIGURA 2:Representação dos fatores geradores de estresse dos profissionais entrevistados que atuam no Centro Cirúrgico em diferentes hospitais de Patos de Minas-MG.

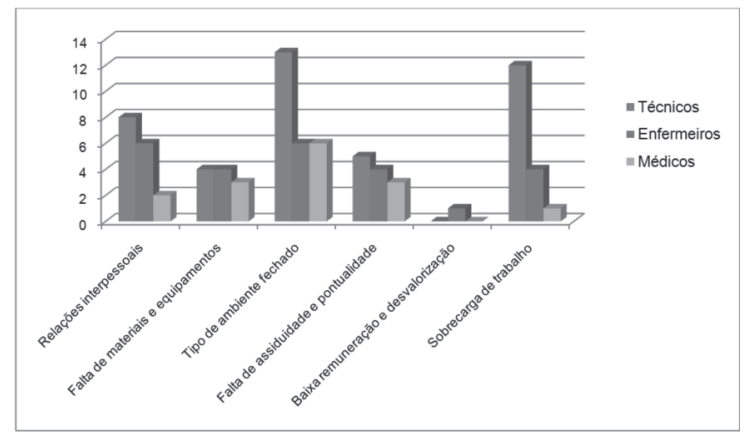




\section{DISCUSSÃO}

Nossos resultados obtidos neste estudo, em relação ao perfil da qualidade de vida dos profissionais que atuam no Centro Cirúrgico (CC) em diferentes hospitais de Patos de Minas-MG, confirmam os achados de outras pesquisas, onde o profissional mais envolvido pertence ao sexo feminino e atua como técnico de enfermagem (COSTA, MORITA e MARTINEZ, 2000; PEREIRA e FÁVERO, 2001). Essas informações reafirmam que tradicionalmente, as funções relacionadas ao cuidado dos pacientes, com suas especialidades tecnológicas próprias de auxiliar, higienizar, alimentar, fornecerem os elementos indispensáveis ao bom desenvolvimento do doente, seguindo os padrões da divisão social do trabalho, sempre esteve confiado ás mulheres (OLER et al.,2005).

Em relação à faixa etária dos profissionais entrevistados, a média de idade foi de 36 anos. Já para a variável estado civil os resultados obtidos apontam que a maioria dos profissionais entrevistados eram casados (77,3\%). É importante destacar que o grupo entrevistado, constitui-se principalmente de profissionais de faixa etária relativamente mais baixa, sendo a maioria casados, o que nos leva a crer que a socialização, isto é, fazer amigos e o relacionamento com a família, reflete na satisfação deste grupo com a dimensão estudada.

Com relação à formação, a proporção de profissionais com $2^{\circ}$ grau completo, superior incompleto e superior completo foi semelhante, $36,4 \%, 27,2 \%$ e $36,4 \%$ respectivamente. No entanto, observou-se que maioria dos profissionais entrevistados recebe remuneração entre $\mathrm{R} \$ 1200,00$ e $\mathrm{R} \$ 1999,00$. Esses dados eram esperados, visto que a maior parte dos entrevistados exerce a função de técnicos de enfermagem. No entanto, no presente estudo, apenas um entrevistado mencionou a baixa remuneração e desvalorização como fator causador de estresse. Assim, embora a baixa remuneração e desvalorização possa causar desmotivação nesses profissionais, acredita-se que os outros fatores sejam mais importantes no sentido que causar estresse dentro do CC.

Após analisar os dados sociodemográficos desses profissionais, avaliamos os fatores que mais causam estresse durante sua atuação no CC, e observamos que o fator que mais causa estresse em ambas as categorias profissionais entrevistadas é o tipo de ambiente fechado, enquanto houve variação dos demais fatoresde acordo com a categoria profissional.É conhecido que o CC é um setor fechado e com particularidades próprias, onde é necessáriolimitar o contato pessoal de fora dessesetor, não ocorrendo, portanto a relação interpessoal com os outros setores dohospital. 0 contato com o ambiente externo geralmente ocorre por via telefônica e por meio do recebimento de materiais e recepção de paciente (SILVA; POPOV, 2010). 
Segundo Passos, Silva e Carvalho (2010), o fato dosprofissionais que atuam CC exercerem as suas atividades funcionais em ambientes fechados, o estresse pode ser um fator presente, considerando que esse setor induz os trabalhadores a se sentirem excluídos de acontecimentos e atividades que ocorram no ambiente hospitalar, como a troca de experiências entre os profissionais e pouca participação em treinamentos levando muitas vezes à defasagem de atualizações sobre a prática nas suas atividades diárias.

Em relação aos técnicos de enfermagem, os outros fatores que mais causam estresse no CC é a sobrecarga de trabalho, relações interpessoais, falta de assiduidade e pontualidade, e falta de materiais e equipamentos respectivamente.Assim, com base nesses resultados é possível observar que além do fato de trabalharem em um ambiente fechado como o CC, a sobrecarga de trabalho e as relações interpessoais é o que mais induz esses profissionais ao estresse.

Quanto aos enfermeiros, outro fator que mais causa estresse no CC são as relações interpessoais, seguidos pela falta de materiais e equipamentos, falta de assiduidade e pontualidade, e sobrecarga de trabalho na mesma proporção, e a baixa remuneração e desvalorização. Diante desses resultados é possível observar que os fatores que mais causam estresse nesses profissionais é trabalhar num ambiente fechado, e as relações interpessoais.Contudo, os outros fatores que mais causam estresse nos médicos dentro do CC é a falta de materiais e equipamentos e a falta de assiduidade e pontualidade na mesma proporção, seguido das relações interpessoais e sobrecarga de trabalho.

Meirelles e Zeitoune (2003), afirmam que algumas características da organização do trabalho hospitalar induzem os profissionais do CC ao estresse, entre elas, as jornadas de trabalho extensas e os ritmos acelerados de trabalho, a conduta repressora e autoritária de uma hierarquia rígida e vertical, a fragmentação das tarefas, os riscos ocupacionais e, consequentemente, de quem o realiza.Estudos relatam que a falta de descanso e de diversão, na maioria das vezes, leva o indivíduo ao estresse que é considerado um problema negativo, de caráter perceptivo, resultante da incapacidade de suportar as pressões no ambiente de trabalho (LENTZ et al., 2000).

A falta de planejamento correto quanto aos procedimentos realizados no CC também pode desencadear conflitos entre a equipe multiprofissional, gerando estresse e impossibilitando a assistência adequada (SILVA; POPOV, 2010). Além disso, a falta de assiduidade e pontualidade de alguns profissionais no CCexige que realizem inúmeras tarefas que deveriam ser repartidas com outros membros da equipe. Isso resulta no aumento das exigências físicas e emocionais, gerando estresse físico e/ou mental e influenciando na qualidade desses profissionais (RODRIGUES, 2012). 
Com relação às relações interpessoais, Monteiro et al. (2013) relatam como origem do estresse e muito desgastante o relacionamento entre a equipe multiprofissional e familiares dos pacientes no CC. Muitas das vezes, os familiares dos pacientes muitas vezes atribuem os erros cirúrgicos aos profissionais responsáveis, pois são eles que passam com o paciente a maior parte do tempo e são os responsáveis pelos cuidados. Nesse contexto, éfundamentalter compreensão e empatia, além de respeitar as diferenças entre os membros da equipe. Quanto aos familiares, responder na medida do possível os questionamentos, minimizando a angústia e sofrimento, pois é o profissional que mantém maior contato e por mais tempo com paciente.

Outros autores afirmaram que os equipamentos e materiais que não funcionam ou que funcionam inadequadamente durante os procedimentos cirúrgicos, utilização de materiais impróprios ou estragados, ausência de material e equipamentos em quantidade insuficiente para os pacientes, uso de produtos inadequados e improvisos, são mencionados como estressores para o grupo cirúrgico, sobrecarregando a atuação do ajudante e causando estresse. Estas situações estressantes interferem significativamenteno equilíbrio emocional do profissional (CAREGNATO; LAUTERT, 2005; SILVA; POPOV, 2010).

Com base nos resultados expostos no presente estudo, seria importante considerar as necessidades individuais destes profissionais, bem como sua aptidão de superação, adaptação e reação nas diferentes situações da vida pessoal, social e profissional, uma vez que cada indivíduo características próprias de lidar com situações conflitantes que desencadeiam o estresse. Inicialmente, os fatores de estresse identificados neste estudo servem de apoio para gerar reflexões acerca da qualidade de vida dos profissionais relacionadas às condições do ambiente de trabalho, implementando programas de atenção à saúde do trabalhador, organização racional e valorização do trabalho, bem como a interação multiprofissional.

\section{CONCLUSÕES}

Diante dos resultados encontrados no presente estudo, conclui-se que os profissionais atuantes no centro cirúrgico em Patos de Minas - MGvivenciaram o estresse relacionado a fatores de natureza objetiva como: as relações interpessoais, falta de materiais e equipamentos, tipo de ambiente fechado, falta de assiduidade e pontualidade, sobrecarga de trabalho, e em menor proporção, a baixa remuneração e desvalorização. 
Embora os objetivos propostos neste estudo foram alcançados, é indispensável considerar os resultados em sua singularidade, uma vez que representa a realidade de uma pequena parte de profissionais atuantes no CC de hospitais do município de Patos de Minas-MG. Novos estudos devem ser realizados afim de identificar os principais fatores que geram estresse nos profissionais que atuam no CC em hospitais de diferentes regiões do país, afim de buscar alternativas para minimizar ou até mesmo elucidar os fatores que interferem na sua qualidade de vida.

\section{REFERÊNCIAS}

CAREGNATO, R. C. A. Estresse da equipe multiprofissional na sala de cirurgia: um estudo de caso. 2002. Tese de Doutorado. Universidade Federal do Rio Grande do Sul. Porto Alegre - RS. Escola de Enfermagem.

CAREGNATO, R. C. A.; LAUTERT, L. 0 estresse da equipe multiprofissional na Sala de Cirurgia. Revista Brasileira de Enfermagem, Brasília, v. 58, n.5, p. 545-50, 2005.

CERVO AL, BERVIAN PA. A pesquisa: noções gerais. 4⿳亠丷a ed. São Paulo: Makron Books; 1996.

COSTA, E. S.; MORITA, I.; MARTINEZ, M. A. Percepção dos efeitos do trabalho em turnos sobre a saúde e a vida social em funcionários da enfermagem em um hospital universitário do Estado de São Paulo. Cadernos de saúde pública, p. 553-555, 2000.

FERRAREZE, M. V. G.; FERREIRA, V.; CARVALHO, A. M. P. Percepção do estresse entre enfermeiros que atuam em Terapia Intensiva. Acta paulenferm, v. 19, n. 3, p. 310-5, 2006.

LENTZ, R. A., COSTENARO, R. G. S., GONÇALVES, L. H., NASSAR, S. M. O profissional de enfermagem e a qualidade de vida: uma abordagem fundamentada nas dimensões propostas por Flanagan. Revista Latino-Americana de Enfermagem, v. 8, n. 4, p. 7 $14,2000$.

MARTINS, F. Z. Atividades gerenciais do enfermeiro em centro cirúrgico. 2013.

MEIRELLES, N. F.; ZEITOUNE, R. C. G. Satisfação no trabalho e fatores de estresse da equipe de enfermagem de um centro cirúrgico oncológico. Escola Anna Nery Revista de Enfermagem, v. 7, n. 1, p. 78-88, 2003.

MIRANDA, E. P. Qualidade de vida de profissionais de enfermagem que atuam em centro cirúrgico. 2006. Dissertação de mestrado. Universidade Federal da 
Paraíba. João Pessoa - PB. Centro de Ciências da Saúde.

MONTEIRO, J. K.;OLIVEIRA, A. L. L.; RIBEIRO, C. S; GRISA, G. H, AGOSTINI, N.Adoecimento psíquico de trabalhadores de unidades de terapia intensiva. Psicologia ciência e profissão, v. 33, n. 2, p. 366-79, 2013.

OLER, F. G., JESUS, A. F. D., BARBOZA, D. B., e DOMINGOS, N. A. M.. Qualidade de vida da equipe de enfermagem do centro cirúrgico. ArqCiênc Saúde, v. 12, n. 2, p. 102-10, 2005.

PASSOS, J. B.; SILVA, E. L.; CARVALHO, M. M. C. Estresse no centro cirúrgico: uma realidade dos profissionais de enfermagem. Revista de Pesquisa em Saúde, v. 11, n. 2, p. 35-8, 2010.

PEREIRA, M. C. A.; FÁVERO, N. A motivação no trabalho da equipe de enfermagem. Revista Latino-Americana de Enfermagem, v. 9, n. 4, p. 7-12, 2001.

RODRIGUES, R. A. P.; SOUSA, F. A. E. F. 0 trabalho da enfermagem em centro cirúrgico: análise de depoimentos. Rev. Latinoam. Enferm, v. 1, n. 2, p. 21-34, 1993.

RODRIGUES, T. D. F. Fatores estressores para a equipe de enfermagem da unidade de terapia intensiva. Revista Mineira de Enfermagem, v. 16, n. 3, p. 454-462, 2012.

SCHMIDT, D. R. C., DANTAS, R. A. S., MARZIALE, M. H. P., E LAUS, A. M. Estresse ocupacional entre profissionais de enfermagem do bloco cirúrgico. Texto \& Contexto-Enfermagem, v. 18, n. 2, p. 330-337, 2009.

SILVA, P. P., POPOV, D. C. S. Estresse da equipe de enfermagem no centro cirúrgico. Revista de Enfermagem UNISA, v. 11, n. 2, p. 125- 30, 2010.

STUMM, E. M. F.; MAÇALAI, R. T.; KIRCHNER, R. M. Dificuldades enfrentadas por enfermeiros em um centro cirúrgico. Texto Contexto Enferm, v.15 n.3 p.464-71, 2006. 